\title{
Study of the thermo-physical properties of bitumen in hydrocarbon condensates
}

\author{
A. Miadonye, J. Cyr, K. Secka \& A. Britten \\ Department of Chemistry Cape Breton University Sydney, Canada
}

\begin{abstract}
The reduction of viscosity with inappropriate diluents could lead to asphaltene precipitation. To use condensates effectively however, their effects on asphaltene precipitation and deposition must be well examined. In this study, the influence of inter-particle interactions of asphaltene on the viscosity and heat of mixing in solutions of bitumen-condensate mixtures are compared to those of bitumen in aromatic solvents and in condensate-toluene mixtures. Three bitumen, two heavy oils, and five condensates from different reservoirs were examined. The results indicate that the presence of aromatic solvent in bitumen delays the on-set of asphaltene precipitation due to the presence of dipole-dipole interactions as well as hetero-molecular interactions. In pure condensates, strong hydrogen bonding and moderate homo-molecular interactions are predominant and, therefore, result in reduced asphaltene precipitation and low enthalpy of mixing. The on-set of asphaltene precipitation in bitumen and heavy oils is delayed in the presence of aromatic solvents, decreasing with reduction in the composition of aromatic solvent in the condensates.
\end{abstract}

Keywords: thermo-physical property, bitumen, hydrocarbon condensates, solvents, asphaltene, calorimetry, viscosity, heavy oil.

\section{Introduction}

Bitumen and heavy oil contain high concentrations of asphaltenes, heteroatoms, and maltenes and possess high densities and viscosities; the characteristics that make their production, transportation and refining very difficult [1,2]. The addition of hydrocarbon diluents promotes the reduction of viscosity and has been employed in several upstream bitumen recovery technologies with some measure of success [3]. Traditionally, producers have used large quantities of 
aromatic solvents in the vapour extraction (Vapex) process to reduce the viscosity of bitumen under reservoir conditions. However, due to environmental concerns, and the high costs of toluene and xylene, the focus has shifted to paraffinic diluents, preferably natural gas condensates. Using low molecular weight paraffinic hydrocarbons in reducing the viscosity of bitumen results in asphaltene precipitation and deposition. This can cause severe clogging of wells and pipelines and totally halt production, leading to a loss in revenue [4]. Asphaltene are defined, according to ASTM standards (ASTM D2007, ASTM D2006, and ASTM D4124), as n-pentane and n-heptane insoluble and toluene soluble petroleum fraction of complex molecular structures.

In the efforts to remediate the problems, calorimetric methods offer valuable information into the heat effects and phase transformations surrounding the asphaltene precipitation. However, it has proven very difficult to provide standard evaluations due to their heteroatom content, complex molecular structure, and polarity [5-7]. Heat is either evolved or absorbed during the creation or destruction of these molecular bonds and thus provides the framework for this study. Prior work in our group addressed aspects of asphaltene threshold multiphase behaviour in pure solvents such as hexane, toluene and different naphtha [8,9]. In this study, different bitumen samples are mixed with various binary mixtures of condensates and toluene. Their heats of mixing are determined. The weight percent of asphaltene precipitation and changes in viscosity at various concentrations are examined with heat of mixing to evaluate changes in the thermo-physical property of the mixtures at the onset of asphaltenes precipitation.

\section{Experimental section}

\subsection{Materials}

Five bitumen, Husky Golden Lake, Celtic, Cold Lake, Plover Lake and Rush Lake, (from Alberta, Canada) supplied by Alberta Research Council; Mongstad light crude oil (from Norway) and naphtha supplied by Esso Refinery (Halifax, Canada); and natural gas condensates (from Sable Island, Nova Scotia, Canada) supplied by Exxon-Mobil Corporation, were selected and used as received for this study. The hydrocarbon solvents (n-pentane, ethylbenzene, toluene and xylenes), and methanol are analytical grade chemicals (Sigma-Aldrich, HPLC grade, $99.9+\%$ ) commercially available and were used as received.

\subsection{Experimental methods}

Asphaltene precipitation was carried out using a 1:40 mL bitumen:n-pentane ratio. The mixture was agitated using a G10 gyratory shaker for 24 hours, left to stand for two hours, and then filtered using a $7.0 \mathrm{~cm}$ Whatman filter paper. The asphaltenes remain as the residue on the filter paper. This was rinsed with an excess of $n$ - pentane until it turned colourless. The precipitated asphaltenes were placed in a desiccator for evaporation of any excess liquid. Masses were 
recorded until a final constant mass was obtained. In a separate experiment, the filtrate which is a mixture of an excess of n-pentane and the deasphalted oil was vacuum distilled to recover the n-pentane and the deasphalted bitumen.

To determine the solubility of bitumen in condensates and toluene, a Parr 1455 solution calorimeter was used to measure the enthalpies of mixing. The calorimeter was interfaced with a desktop PC for automatic data acquisition and analysis. The calorimeter's Dewar unit is equipped with a microprocessor-based thermometer with a 0.0008 heat leak constant when measuring results obtained at atmospheric pressure and a $+/-5^{\circ} \mathrm{C}$ of room temperature.

The calorimeter was standardized with tris[hydroxymethyl]aminomethane (THAM) and $0.1 \mathrm{M}$ hydrochloric acid in accordance with the procedure specified in the manufacturer's manual. To measure the enthalpy of mixing, masses ranging from $0-20 \mathrm{~g}$ of the pure bitumen or bitumen-diluent mixtures were placed in a rotating sealed glass sample cell inside the Dewar (solvent chamber). Thermal equilibrium is allowed to be reached before the sample is then released into the solvent chamber. This mixture will reach a new equilibrium temperature as the reaction is complete; thus producing the thermogram. As depicted in Figure 1, the thermogram provides the initial and final temperatures which are obtained through the trend line equations drawn from temperatures before and after mixing period. The heat values and hence the enthalpy of mixing are calculated in accordance with the procedure and equation given in PARR's manufacturer manual.

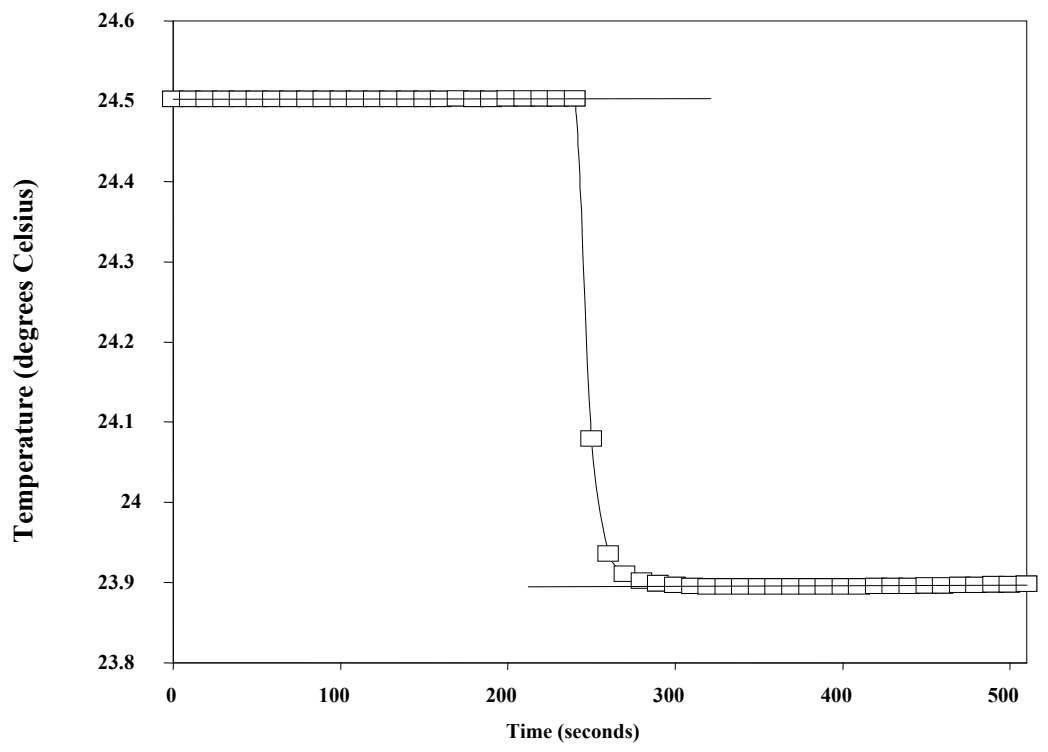

Figure 1: Typical solution calorimetry thermograph for mixing solvent with heavy petroleum blend. 


\section{Results and discussion}

The results obtained for the thermo-physical property transformations when bitumen is mixed with light oil, condensates and naphtha are illustrated in Figures 2 to 6. Enthalpies of mixing for pure Mongstad light oil, and for a 50/50 w/w mixture of Rush Lake bitumen with the Mongstad oil are shown in Figure 2.

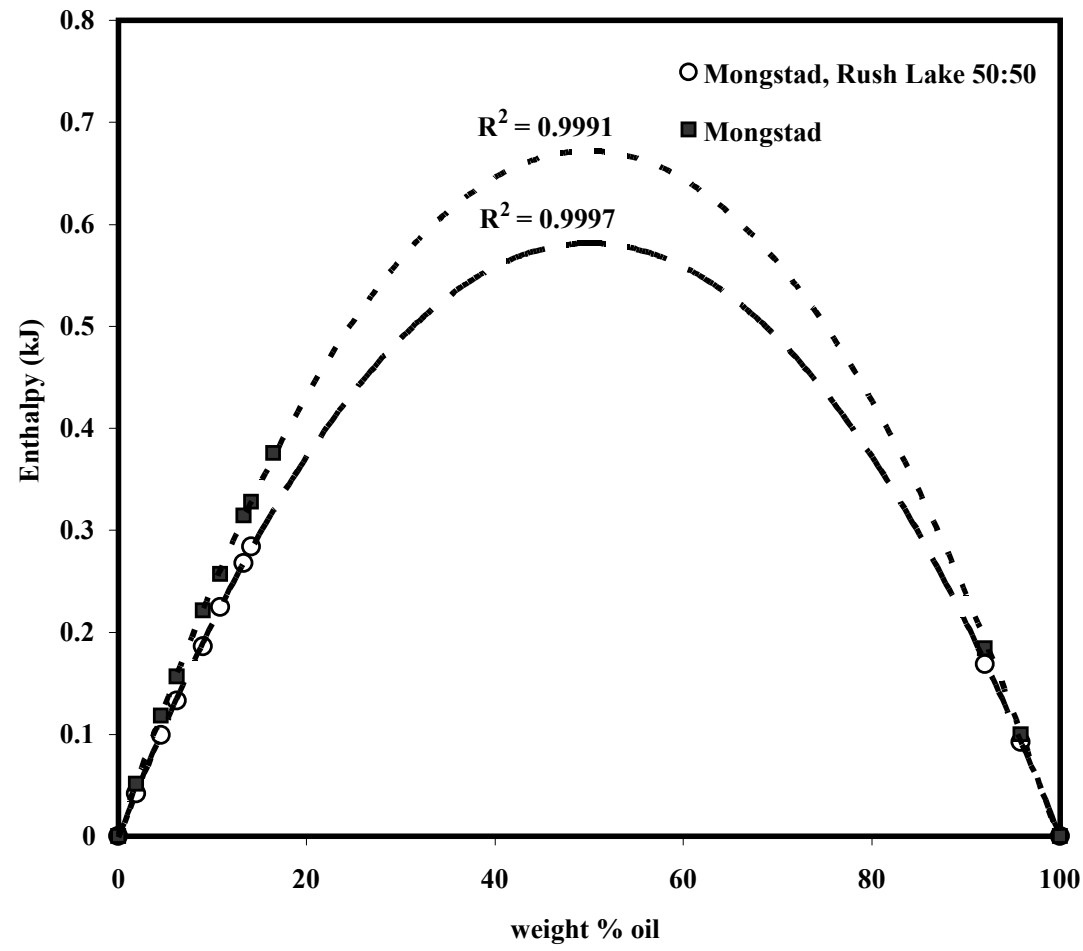

Figure 2: $\quad$ Enthalpy of mixing for pure light oil and 50:50 w/w Rush Lake bitumen and light oil mixture in toluene.

The trend lines gave parabola patterns of endothermic nature and it is observed that the presence of Rush Lake in the Mongstad oil causes the values of enthalpy to decrease by shifting the parabola closer to the $\mathrm{x}$-axis. This suggests that the Rush Lake bitumen present in the sample retains the energy which would otherwise be used to mix the bitumen with the toluene. Figure 3 shows the enthalpies of mixing at lower concentrations of bitumen and bitumen blends in $100 \mathrm{~mL}$ toluene. It is observed that the presence of Rush Lake causes the values of enthalpy to decrease. Therefore, the presence of Rush Lake removes more heat from the condensates-toluene mixture, absorbing the heat normally dispersed from mixing pure condensates with toluene. The results indicate that 
the higher the mass of bitumen in the ratio of bitumen-condensates mixtures, the lower the enthalpy of mixing. It takes more energy to dissolve the large molecules of bitumen during the mixing. This is evident by the fact that replacing the condensates with higher viscosity Mongstad light oil further reduced the heat of mixing.

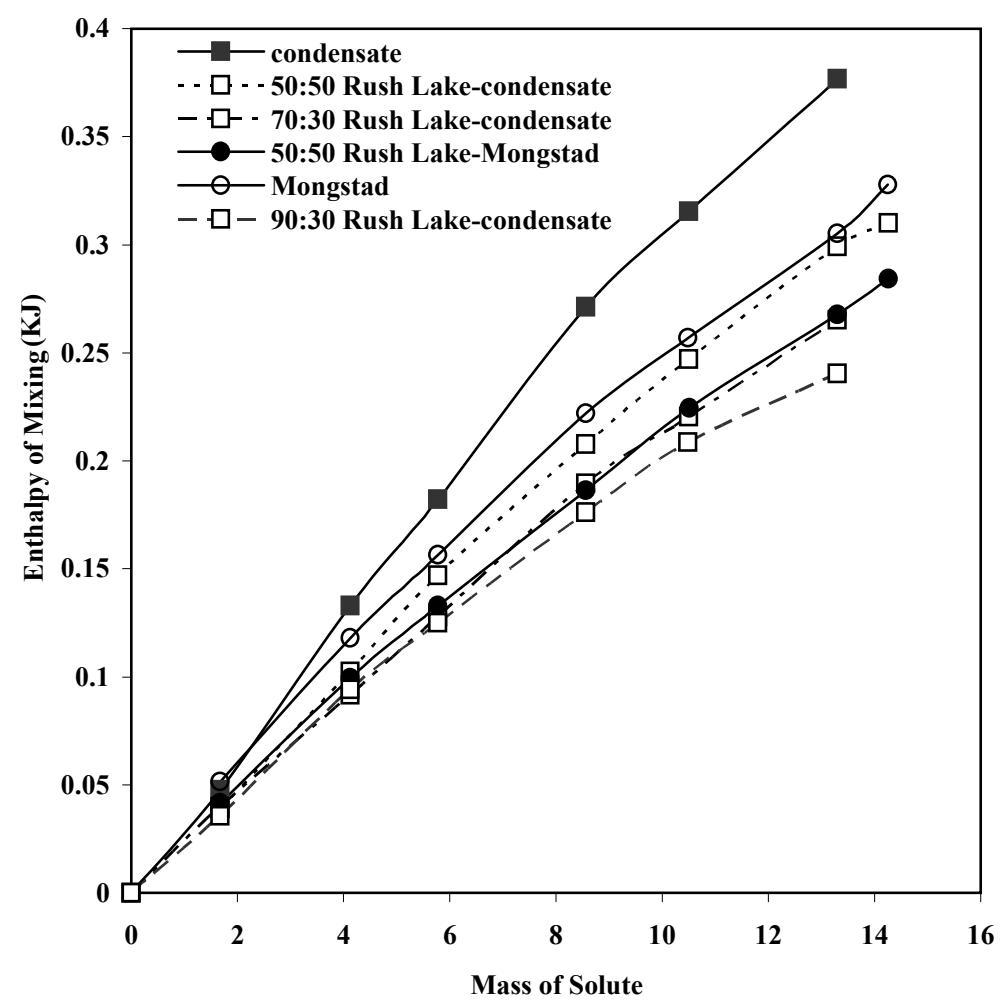

Figure 3: Enthalpy of mixing for pure diluents and different compositions of bitumen and diluents.

When the asphaltene from the samples were recovered and weighed, it was observed that the amounts of asphaltene in the mixtures were inversely proportional to the per cent diluents. Figure 4 depicts the enthalpies of mixing for asphalted and deasphalted bitumen. It is suggested from the results that the presence of bitumen in the mixture withholds the energy usually released upon mixing. This energy is contained within the molecular components in the bitumen, with lower enthalpies of mixing for asphalted bitumen than for deasphalted bitumen.

The de-asphalted bitumen and the asphalted bitumen were each made into solutions containing $10 \mathrm{~g}$ of the bitumen with $20 \mathrm{~mL}$ of toluene. The viscosities of each were determined to see the effect of asphaltene on the viscosity. The 


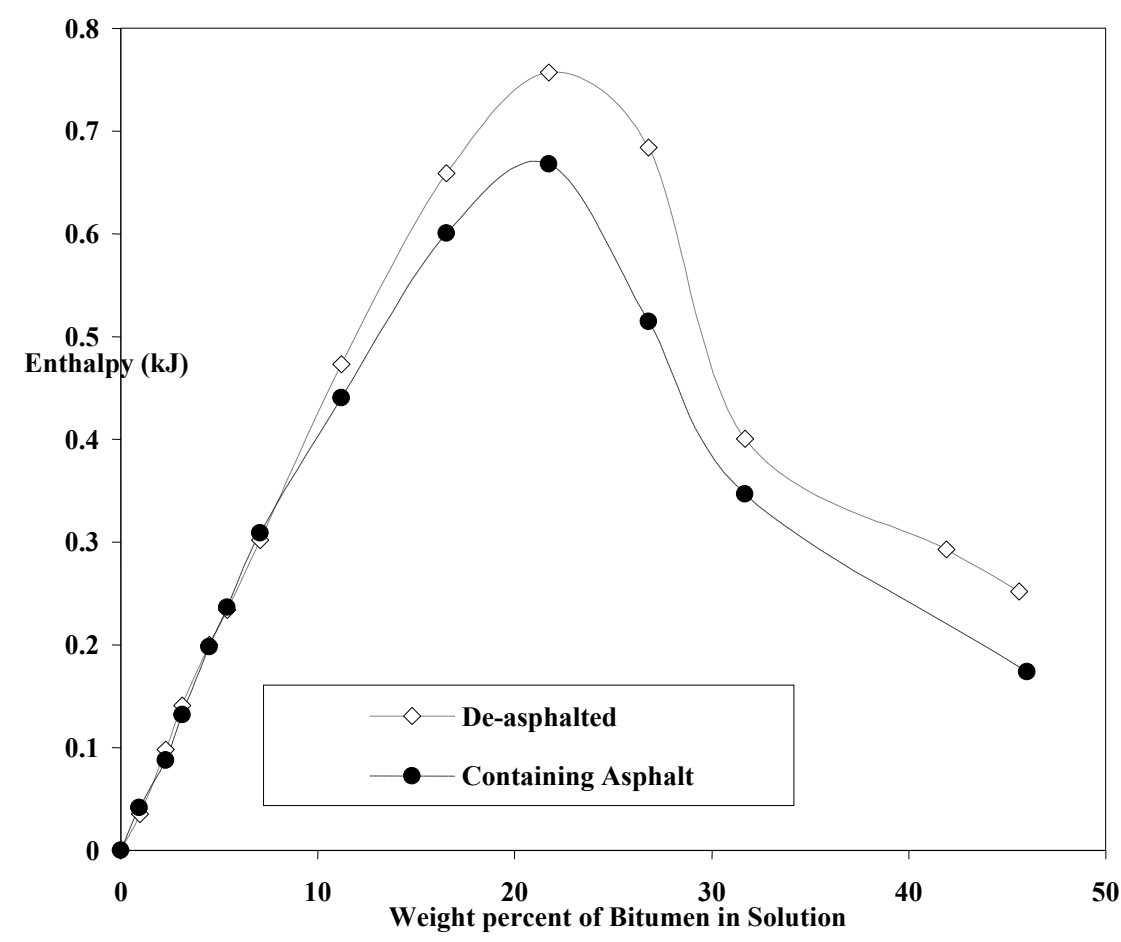

Figure 4: Enthalpy of mixing for 50:50 w/w Plover lake bitumen: condensate mixture in toluene.

original bitumen mixtures (in Figures 5 and 8) show large reductions in viscosity with increase in weight percent of condensates. The large reduction in viscosity at 50:50 w/w ratio compared to blends with larger ratios of bitumen is not observed for enthalpies of mixing. This observation indicates that asphaltene alone is not responsible for the endothermic property of the mixture. In Figure 6, the effect of solvent type on solubility of bitumen is shown for Cold Lake bitumen. For the same bitumen and similar volume per cent of solvents, bitumen mixed with toluene showed lower viscosities at different temperatures compared to bitumen-naphtha mixtures. Aromatic solvents such as toluene have been shown to be good solvents for bitumen as they dissolve asphaltene $[1,4,8,9]$.

As noted in the Introduction, asphaltene are defined as toluene soluble, the solubility in toluene could well be attributed to the polar nature of asphaltene. Consequently, n-paraffin with no inherent dipolar molecules will increase hetero-molecular interactions, leading to asphaltene precipitation in high solvent concentration.

To examine further the mixing characteristics of bitumen and condensates, the enthalpies of mixing for low molecular weight paraffin and aromatic 


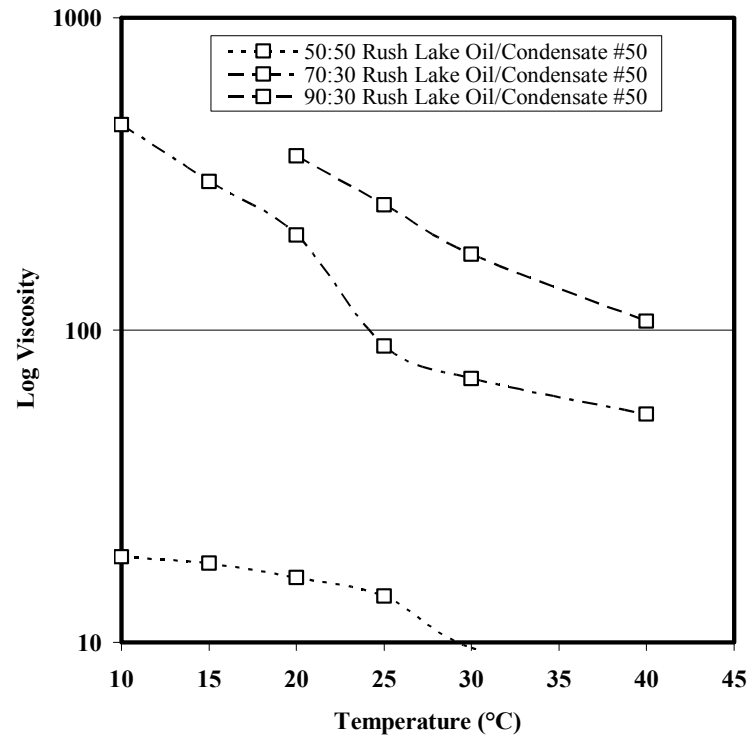

Figure 5: Viscosity-temperature relationship for different bitumencondensate mixtures.

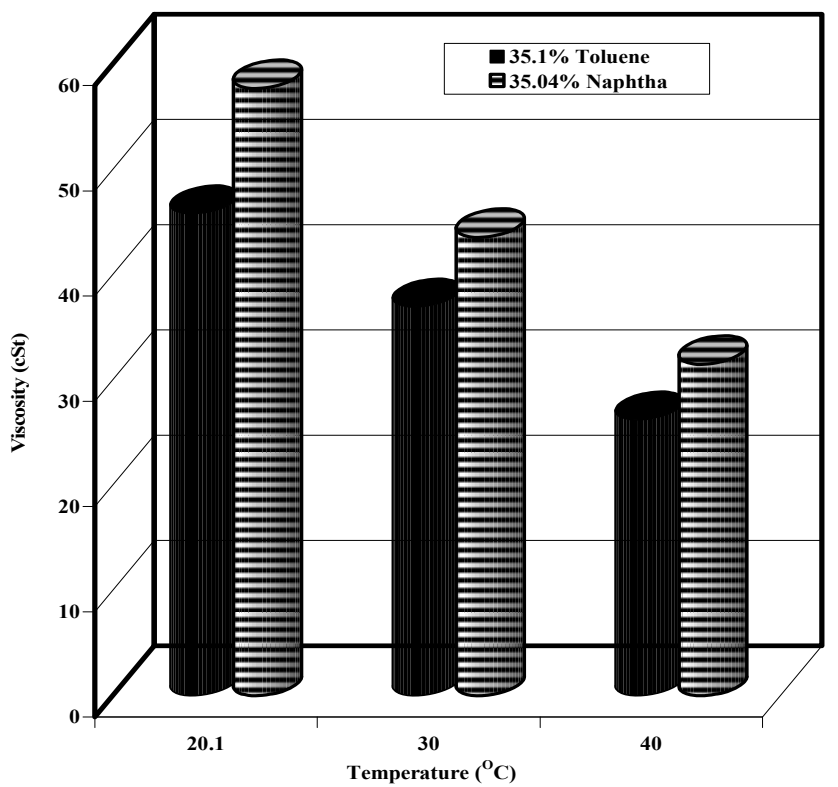

Figure 6: Effects of solubility and temperature on the viscosity of Cold Lake bitumen. 


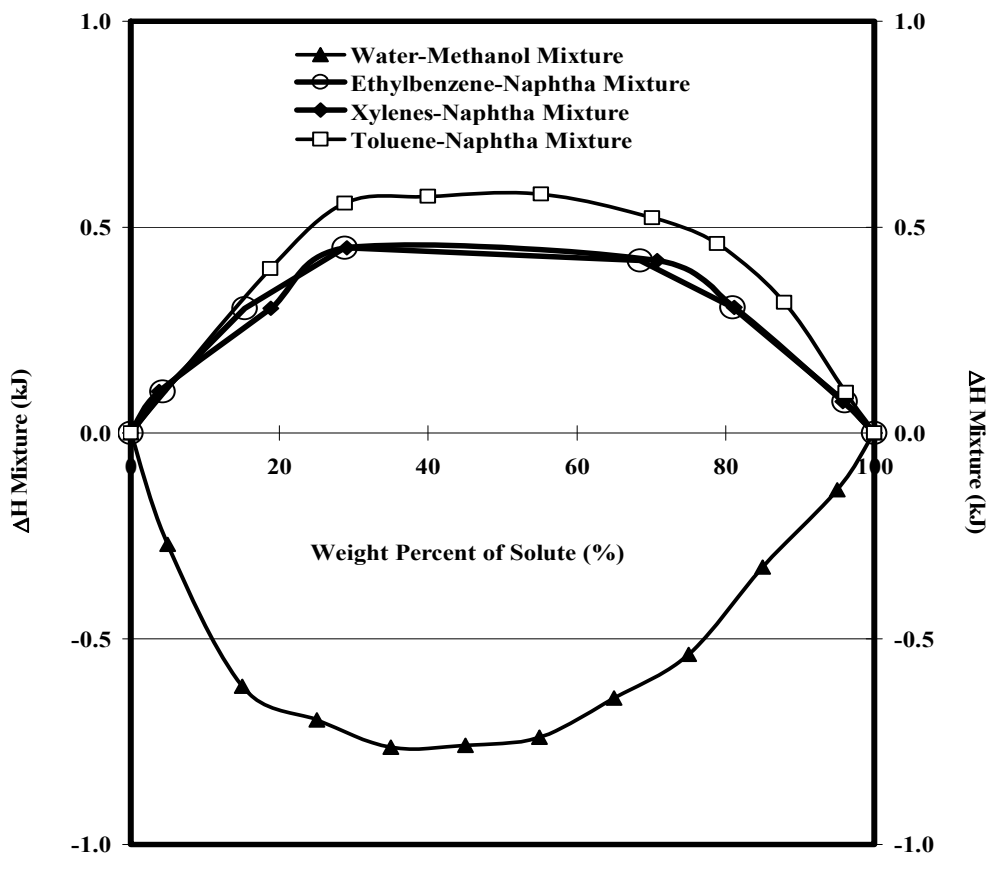

Figure 7: Enthalpy of mixing for several binary systems with a high level of miscibility.

solvents, and for methanol-water systems, were determined as illustrated in Figure 7. The slope of enthalpy versus volume percent of methanol decreases with increasing concentrations showing that the enthalpies of mixing distilled water with methanol are exothermic. The result represented in Figure 7 is a well known standard enthalpy diagram for methanol-water mixture. In contrast, the non-polar hydrocarbon solvents exhibited endothermic enthalpies of mixing. The toluene-naphtha mixture is more endothermic than ethylbenzene-naphtha and xylene-naphtha mixtures. This observation is in agreement with results obtained for bitumen-condensates mixtures. Both ethylbenzene and xylene are slightly larger molecules than toluene and their enthalpies of mixing are lower. This suggests that molecular size, and possibly structure, affect heat of mixing. Previous work showed that this argument is true for hydrocarbon solvent mixtures [8]. The presence of extra methyl pendent groups on the aromatic solvent (ethylbenzene and xylene) allows for greater homomolecular interactions with naphtha resulting in lower enthalpy of mixing.

\section{Conclusions}

The enthalpy of mixing and phase transformations of bitumen in paraffinic diluents such as natural gas condensates and naphtha are reduced with addition of aromatic solvent due to the occurrence of dipole-dipole interactions. Low 
enthalpy of mixing and reduced asphaltene precipitation is observed for bitumenlight oil mixtures illustrating the influence of hydrogen bonding and homomolecular interactions in bitumen solubility. These results also show clearly that bitumen blend is stable in low concentrations of condensates (up to 5 per cent) but for higher concentration of condensates would require addition of aromatic solvent additive to prevent or reduce the precipitation of asphaltene.

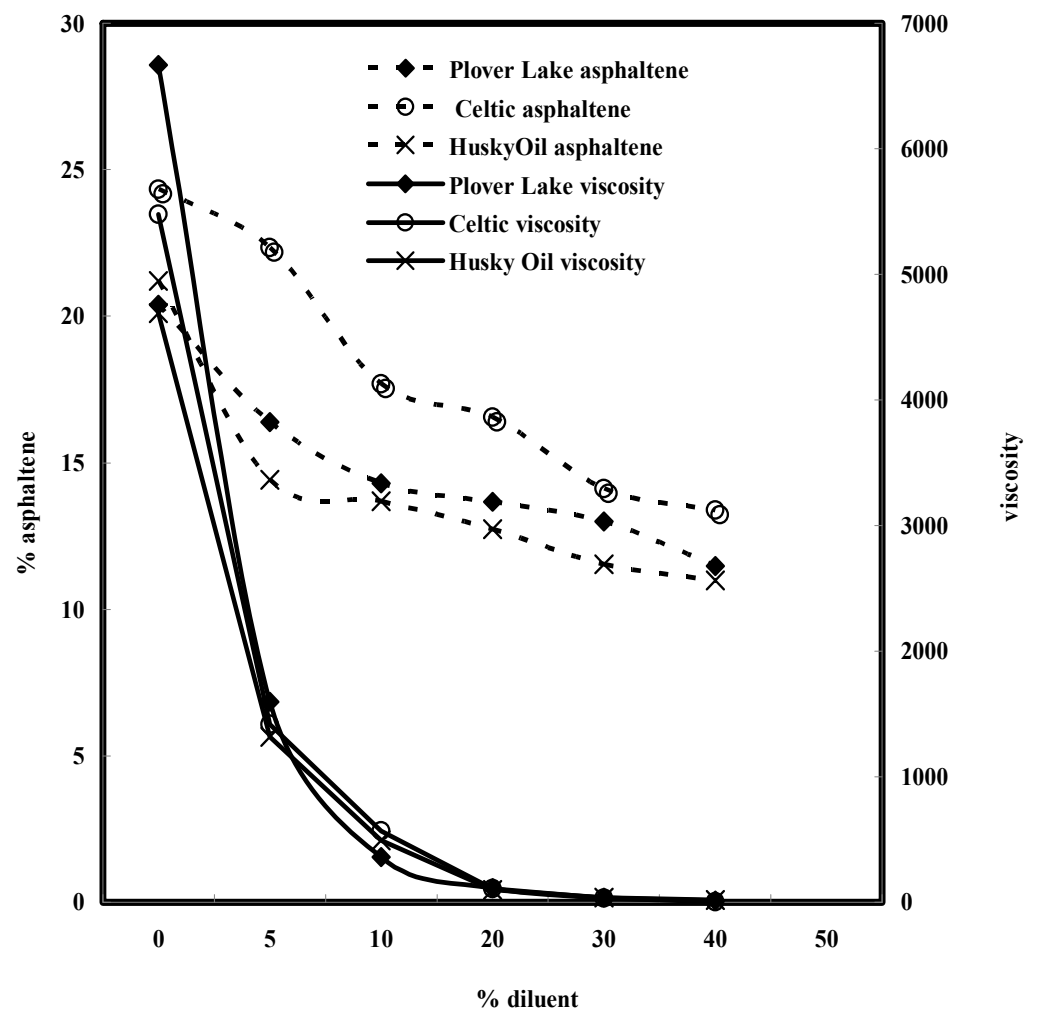

Figure 8: Comparison of changes in asphaltene content and viscosity with condensate composition for different bitumens.

\section{Acknowledgements}

This work was funded by an NSERC-undergraduate student research award (USRA). The support of CBU-ORAI is highly appreciated. Thanks to Loree D’Orsay for her data analysis contributions.

\section{References}

[1] Reynolds, J.G., Metals and heteroatoms in heavy oils. Petroleum Chemistry and Refining, ed. J.G. Speight, Taylor and Francis: Washington DC, 1999. 
[2] Speight, J.G., Petroleum analysis and evaluation. Petroleum Chemistry and Refining, ed. J.G. Speight, Taylor and Francis: Washington DC, 1999.

[3] Miadonye, A., Singh, B., Huang, S.S., Srivastava, R. \& Puttagunta, V.R., Modelling the Effect of Dissolved Gases on the Viscosity of Heavy Oils. Chemical Engineering Research and Design, 73 (A2), pp. 208-213, 1995.

[4] Das, S.K., \& Bulter, R.M., Mechanism of the vapour extraction process for heavy oil and bitumen. Journal of Petroleum Science, 12, pp. 219-231, 1995.

[5] Porte, G., Zhou, H. \& Lazzeri, V., Reversible Description of Asphaltene Colloidal Association and Precipitation, Langmui. 19, pp. 40-47, 2003.

[6] Alboudwarej, H., Beck, J., Svrcek, W.Y., Yarronton, H.W. \& Akbarzadeh, K., Sensitivity of Asphaltene Properties to Separation Techniques. Energy \& Fuels, 16, pp. 462-469, 2002.

[7] Akbarzadeh, K., Alboudwarej, H., Svrcek, W.Y. \& Yarronton, H.W., A Generalized Regular Solution Model for asphaltene precipitation from $n$ alkane diluted heavy oils and bitumens. Fluid Phase Equilibria, 232, pp. 129-140, 2005.

[8] Miadonye, A., Evans, L. \& McKenna, T.M., Study of Asphaltene Precipitation in Dilute Solution by Calorimetry. Computational Methods in Multiphase Flow III, eds. A.A. Mammoli and C.A. Brebbia, WIT Press, 50, pp.13-21, 2005.

[9] Wallace, D., Henry, D., Miadonye, A. \& Puttagunta, V.R., Viscosity and Solubility of Mixtures of Bitumen and Solvent. Fuel Science \& Technology International, 14 (3), pp. 465-478, 1996. 